\title{
Scheduling of irrigation with mulch under different sugarcane planting methods
}

\author{
Thimmegowda Puttavenkategowdal*, and T. E. Nagaraja2 \\ 1Zonal Agricultural Research Station, V. C. Farm, Mandya - 571 405, University of Agricultural Sciences, Bangalore, India \\ 2AICRP on Pigeon pea, GKVK, Bangalore - 560 065, Karnataka, India
}

\begin{abstract}
A filed experiment was conducted at ZARS, V. C. Farm, Mandya to enhance the crop and water productivity in sugarcane during 2017-18 and 2018-19. The design used was strip plot design with planting methods as a horizontal factor and irrigation scheduling as a vertical factor with three replications. Among the planting methods, $120 \mathrm{~cm}$ row spaced furrow planting with dhaincha green manure sowing at 30 DAP and mulching at 75 DAP recorded significantly higher cane yield (158.5 and $161.0 \mathrm{t} / \mathrm{ha}$, respectively) as compared to others during both the years of experimentation. Among the irrigation schedules, IW/CPE ratio of 1.0 recorded significantly higher yield (168.1 and $170.8 \mathrm{t} / \mathrm{ha}$, respectively) as compared to IW/CPE ratio of 0.60 (129.7 and $131.8 \mathrm{t} / \mathrm{ha}$, respectively). The total water used was significantly lower in $120 \mathrm{~cm}$ spaced furrow planting with alternate skip furrow irrigation afte $5 \mathrm{r}$ earthing + green manure mulching (1773 and $1751 \mathrm{~mm}$, respectively) and it saved $16 \%$ of irrigation water. Scheduling of irrigation at IW/CPE ratio of 0.6 consumed lowest amount of irrigation water $(1725 \mathrm{~mm})$ and saved $18 \%$ of irrigation water.
\end{abstract}

\section{INTRODUCTION}

Sugarcane is one of the important tropical crops and requires warm humid climate for efficient solar-energy harvest and to produce 1 tonne sugarcane, the crop needs 125 tonnes water [4]. There is a linear relationship between the growth rate and optimum soil moisture because only vegetative growth is of economic importance [10]. Sugarcane being one year crop has high water requirement than other crops because of long tillering phase during summer months when there is higher evaporative demand. Formative phase of the crop, in which the crop remains young and tender, coincides with hot and desiccating summer and hence, optimum soil moisture is of paramount important to get good yields. [6] Reported that only $35 \%$ of the total area under sugarcane receives optimum irrigation and remaining $65 \%$ area is under sub-optimum irrigation or un-irrigated. In tropical India, the number of irrigations range from 30 to 36; while in the subtropics 5 to 10 irrigations are required with a depth of $80 \mathrm{~mm}$. The water requirement of sugarcane in India varies widely from 1,143 to $3,048 \mathrm{~mm}$ [3]. Scientists have worked to enhance irrigation water-use efficiency $1.5-2.5$ times through devising advance irrigation methods or modifying existing surface-irrigation methods. It is generally accepted that adoption of irrigation in a scientific manner by farmers is far below the expectations and farmers need more comprehensive technological support that is simpler to use and can be integrated into farm management. Non-optimum yields of sugarcane are obtained by maintaining very high moisture in entire rhizosphere

during the entire growing season, until about 1 month before harvesting. Thus, irrigation scheduling based on climato- logical approach is need of the hour for increasing water use efficiency in sugarcane. Irrigation scheduling based on pan evaporation reduces the irrigation requirement without any adverse effect on the yields. The major advantage of this approach is that farmers need not change the amount of water applied from one irrigation to another and the event of rain also taking care off. Irrigation based on this approach permits the computation of a timetable for the irrigation, provided the pattern of pan evaporation does not show much yearly variation during the growing period [8]. Over the period, different planting methods have been developed for improving cane productivity and water use efficiency. In the past, many researchers reported higher water use efficiency in sugarcane with techniques like skip furrow method of irrigation, irrigation at critical growth stages, trash mulching, growing of mulch crops in between rows and ring pit method of planting. Irrigation water use efficiency in sugarcane with these water saving technologies can be enhanced by 1.5 to 2.5 times. In tropical India alternate skip furrow irrigation along with mulching of green manure crops is found to enhance the water use efficiency without reduction in the cane yield. Keeping these in view, the present field experiment was conducted to optimize irrigation schedule in sugarcane under different planting methods.

\footnotetext{
*Corresponding author: thimmegowdap $@$ gmail.com
} 


\section{MATERIAL AND METHODS}

A field experiment was conducted at Zonal agricultural Research Station, V. C. Farm, Mandya during 2017-18 and 2018-19. The initial soil analysis of the experimental site indicated that, the soil was low in organic carbon $(0.39 \%)$, neutral in $\mathrm{pH}(6.70)$, low in available nitrogen (220 kg/ha), available P2O5 (18.5 kg/ha) and available $\mathrm{K} 2 \mathrm{O}(130 \mathrm{~kg} / \mathrm{ha})$. The design adopted was strip plot with planting methods as a horizontal factor (P1: Furrow planting (120 cm row spacing) without mulching; P2: Furrow planting (120 cm row spacing) with green manure (dhaincha) sowing at $30 \mathrm{DAP}$, mulching at 75 DAP and earthing-up at 110 DAP; P3: Furrow planting $(120 \mathrm{~cm}$ row spacing) with alternate skip furrow irrigation after earthing-up without mulching; and P4: Furrow planting (120 cm row spacing) with alternate skip furrow irrigation after earthing-up + green manure) and irrigation scheduling as a vertical factor (IW/CPE ratio of $0.6,0.8$ and 1.0 ) with three replications. The sugarcane cultivar Co 86032 was planted on 23 and 25th of January during 2017-18 and 2018-19, respectively. The recommended quantity of N, P2O5 and K20 (250: 100: $125 \mathrm{~kg} / \mathrm{ha}$ ) were applied to the crop in the form of urea, single super phosphate and muriate of potash. The entire quantity of P2O5 and K20 and $10 \%$ of the recommended $\mathrm{N}$ were applied as a basal dose before planting. Remaining 20, 30 and $40 \%$ of the recommended $\mathrm{N}$ was applied as top dress at 1.5, 2.5 and 3.5 months after transplanting. The spacing followed was $120 \mathrm{~cm}$ between rows and $60 \mathrm{~cm}$ between plants. The green manure crop dhaincha (Sesbania acculata) was sown at 30 DAP and mulched at 75 DAP by spraying 2,4 -D. To compute irrigation water applied, water head was measured on parshall flume installed at start of water channel and time was noted to fill the experimental units to the pre-marked levels. Total water applied during whole crop growing season was calculated by cumulating the irrigation-wise depth of water delivered and number of irrigation applied. Cane yield was recorded after topping and trash stripping given as $\mathrm{t} / \mathrm{ha}$. Five millable canes were taken at random from each plot for determining cane length and cane thickness. Juice-quality analysis was done after weighing and crushing through 3 roller miller. A sub sample of crushed cane juice was analyzed for Brix (total solids) by a hand refractrometer. Commercial cane sugar \% (CCS) and sugar yield (CCS) was calculated as per [7].

Commercial cane sugar $(\%)=[S-(B-S) 0.4] \times 0.73(1)$ where $\mathrm{S}=$ sucrose per cent juice $($ pol $\%)$ and $\mathrm{B}=\mathrm{Brix}$

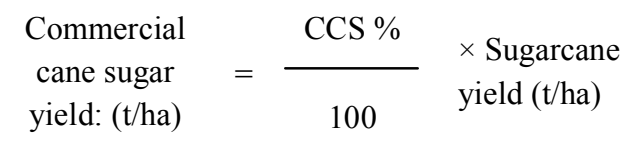

\section{RESULTS AND DISCUSSION}

\subsection{Planting methods}

Planting methods had significant influence on cane yield and yield parameters (Table 1). Among the planting methods, $120 \mathrm{~cm}$ row spaced furrow planting with dhaincha green manure sowing at $30 \mathrm{DAP}$ and mulching at 75 DAP recorded significantly higher cane yield (158.5 and $161.0 \mathrm{t} / \mathrm{ha}$, respectively) as compared to others during both the years of experimentation. However, it was at par with $120 \mathrm{~cm}$ row spaced furrow planting with alternate skip furrow irrigation after earthing up + dhaincha green manure mulching (152.3 and $155.2 \mathrm{t} / \mathrm{ha}$, respectively). This increased cane yield in above treatments was mainly attributed to enhanced yield attributes viz., single cane weight, cane length and millable cane $\mathrm{ha}^{-1}$. Among the planting methods, $120 \mathrm{~cm}$ row spaced furrow planting with dhaincha green manure sowing at 30 DAP and mulching at 75 DAP recorded significantly higher single cane weight ( 2.05 and $2.10 \mathrm{~kg}$, respectively), cane length (2.36 and $2.14 \mathrm{~cm}$, respectively) and millable cane ha-1(84.99 and 85.87 ' $000 /$ ha, respectively) as compared to others during both the years of experimentation. However, it was at par with $120 \mathrm{~cm}$ row spaced furrow planting with alternate skip furrow irrigation after earthing up + dhaincha green manure mulching with respect to single cane weight (1.76 and $1.77 \mathrm{~kg}$, respectively), cane length $(1.96$ and $1.97 \mathrm{~cm}$, respectively) and number of millable cane ha ${ }^{-1}$ (80.84 and $\left.81.73^{\prime} 000 / \mathrm{ha}\right)$. This increased cane yield and yield attributes was mainly due to increased availability soil moisture throughout the crop growing period due to mulching of soil with green manures. According to [9] mulching is beneficial to cane cultivation in dry months and during earthening up. It limits the water losses and soil erosion over the surface of the field [1]. In this manner it plays a positive role in water conservation and the suppression of evaporation which has supplementary effect. It also prevents the rise of water containing salt, which is important in countries with high salt content water resources [2]. Hence, it suppressed weed germination and retarded growth and development of many weeds [11]. They also indicated that mulching also reduced soil erosion and conserved soil moisture. While, the lower cane yield was noticed in $120 \mathrm{~cm}$ row spaced furrow planting with alternate skip furrow irrigation without mulching $(145.3 \mathrm{t} / \mathrm{ha})$ due to shortage of water especially during grand growth stages resulted in reduced single cane weight, cane length and number of millable canes and finally cane yield.

Table 1. Sugarcane growth and yield attributes and yield as influenced planting methods and irrigation schedules.

\begin{tabular}{|c|c|c|c|c|c|c|c|c|}
\hline \multirow{2}{*}{$\begin{array}{l}\text { Treat } \\
\text { ment }\end{array}$} & \multicolumn{2}{|c|}{$\begin{array}{c}\text { Single } \\
\text { cane } \\
\text { weight } \\
\text { (kg) }\end{array}$} & \multicolumn{2}{|c|}{$\begin{array}{c}\text { Cane } \\
\text { length } \\
(\mathrm{cm})\end{array}$} & \multicolumn{2}{|c|}{$\begin{array}{c}\text { Millable } \\
\text { cane } \\
\text { ('000/ha) }\end{array}$} & \multicolumn{2}{|c|}{$\begin{array}{l}\text { Cane } \\
\text { yield } \\
\text { (t/ha) }\end{array}$} \\
\hline & $\begin{array}{c}2017 \\
-18\end{array}$ & $\begin{array}{c}2018 \\
-19\end{array}$ & $\begin{array}{c}20 \\
17- \\
18 \\
\end{array}$ & $\begin{array}{c}201 \\
8-19\end{array}$ & $\begin{array}{c}201 \\
7-18\end{array}$ & $\begin{array}{c}201 \\
8-19\end{array}$ & $\begin{array}{c}201 \\
7-18\end{array}$ & $\begin{array}{c}201 \\
8-19\end{array}$ \\
\hline \multicolumn{9}{|c|}{ Planting methods } \\
\hline $\mathrm{P}_{1}$ & 1. 92 & 1.93 & $\begin{array}{c}2.2 \\
5\end{array}$ & 2.30 & $\begin{array}{l}82 . \\
23\end{array}$ & $\begin{array}{c}82 . \\
81\end{array}$ & $\begin{array}{c}148 \\
.8\end{array}$ & $\begin{array}{c}149 \\
4\end{array}$ \\
\hline
\end{tabular}




\begin{tabular}{|c|c|c|c|c|c|c|c|c|}
\hline $\mathrm{P}_{2}$ & 2.05 & 2.10 & $\begin{array}{c}2.3 \\
6\end{array}$ & 2.41 & $\begin{array}{c}84 . \\
99\end{array}$ & $\begin{array}{c}85 . \\
87\end{array}$ & $\begin{array}{c}158 \\
.5\end{array}$ & $\begin{array}{c}161 . \\
0\end{array}$ \\
\hline $\mathrm{P}_{3}$ & 1.63 & 1.64 & $\begin{array}{c}1.9 \\
6\end{array}$ & 1.97 & $\begin{array}{c}79 . \\
56\end{array}$ & $\begin{array}{c}80 . \\
78\end{array}$ & $\begin{array}{c}143 \\
.1\end{array}$ & $\begin{array}{c}145 . \\
3\end{array}$ \\
\hline $\mathrm{P}_{4}$ & 1.76 & 1.77 & $\begin{array}{c}2.1 \\
1\end{array}$ & 2.13 & $\begin{array}{c}80 . \\
84\end{array}$ & $\begin{array}{c}81 . \\
73\end{array}$ & $\begin{array}{c}152 \\
.3\end{array}$ & $\begin{array}{c}155 . \\
2\end{array}$ \\
\hline $\begin{array}{c}\mathrm{S} . \mathrm{Em} . \\
\pm\end{array}$ & 0.04 & 0.04 & $\begin{array}{c}0.0 \\
3\end{array}$ & 0.04 & $\begin{array}{c}0 . \\
52\end{array}$ & 0.55 & $\begin{array}{c}2 . \\
89\end{array}$ & 2.36 \\
\hline $\begin{array}{c}\mathrm{CD} \\
\text { a 5\% }\end{array}$ & 0.12 & 0.14 & $\begin{array}{c}0.1 \\
2\end{array}$ & 0.13 & $\begin{array}{c}1 . \\
80\end{array}$ & 1.91 & $\begin{array}{c}9 . \\
99\end{array}$ & 8.18 \\
\hline
\end{tabular}

\section{Scheduling of irrigation}

\begin{tabular}{|c|c|c|c|c|c|c|c|c|}
\hline $\mathrm{I}_{1}$ & 1.65 & 1.66 & $\begin{array}{c}2 . \\
00\end{array}$ & 2.03 & $\begin{array}{c}78 . \\
17\end{array}$ & $\begin{array}{c}79 . \\
33\end{array}$ & $\begin{array}{c}129 . \\
7\end{array}$ & $\begin{array}{c}131 . \\
8\end{array}$ \\
\hline $\mathrm{I}_{2}$ & 1.84 & 1.85 & $\begin{array}{c}2 . \\
18\end{array}$ & 2.20 & $\begin{array}{c}82 . \\
96\end{array}$ & $\begin{array}{c}83 . \\
55\end{array}$ & $\begin{array}{c}157 . \\
2\end{array}$ & $\begin{array}{c}155 . \\
6\end{array}$ \\
\hline $\mathrm{I}_{3}$ & 2.03 & 2.07 & $\begin{array}{c}2 . \\
33\end{array}$ & 2.37 & $\begin{array}{c}84.5 \\
9\end{array}$ & $\begin{array}{c}85.5 \\
1\end{array}$ & $\begin{array}{c}168 . \\
1\end{array}$ & $\begin{array}{c}170 . \\
8\end{array}$ \\
\hline $\begin{array}{c}\mathrm{S} . \mathrm{Em} \\
. \pm\end{array}$ & 0.03 & 0.02 & $\begin{array}{c}0 . \\
04\end{array}$ & 0.06 & 1.12 & 1.30 & 3.40 & 3.06 \\
\hline $\begin{array}{c}\mathrm{CD} \\
@ \\
5 \%\end{array}$ & 0.10 & 0.09 & $\begin{array}{c}0 . \\
16\end{array}$ & 0.24 & 4.40 & 5.10 & $\begin{array}{c}13 . \\
35\end{array}$ & $\begin{array}{c}12 . \\
01\end{array}$ \\
\hline
\end{tabular}

\section{Planting methods X Irrigation Scheduling}

\begin{tabular}{|c|c|c|c|c|c|c|c|c|}
\hline $\mathrm{P}_{1} \mathrm{I}_{1}$ & 1.73 & 1.68 & $\begin{array}{c}2 . \\
09\end{array}$ & 2.12 & $\begin{array}{c}78 . \\
57\end{array}$ & $\begin{array}{c}79 \\
33\end{array}$ & $\begin{array}{c}128 . \\
4\end{array}$ & $\begin{array}{c}129 . \\
5\end{array}$ \\
\hline $\mathrm{P}_{1} \mathrm{I}_{2}$ & 1.93 & 1.96 & $\begin{array}{c}2 . \\
27\end{array}$ & 2.34 & $\begin{array}{c}83 . \\
29\end{array}$ & $\begin{array}{c}83 . \\
56\end{array}$ & $\begin{array}{c}151 . \\
6\end{array}$ & $\begin{array}{c}150 . \\
5\end{array}$ \\
\hline $\mathrm{P}_{1} \mathrm{I}_{3}$ & 2.09 & 2.14 & $\begin{array}{c}2 . \\
40\end{array}$ & 2.43 & $\begin{array}{c}84 . \\
84\end{array}$ & $\begin{array}{c}85 \\
52\end{array}$ & $\begin{array}{c}166 . \\
3\end{array}$ & $\begin{array}{c}168 . \\
1\end{array}$ \\
\hline $\mathrm{P}_{2} \mathrm{I}_{1}$ & 1.90 & 1.94 & $\begin{array}{c}2 . \\
16\end{array}$ & 2.21 & $\begin{array}{c}80 . \\
48\end{array}$ & $\begin{array}{c}81 . \\
44\end{array}$ & $\begin{array}{c}135 . \\
5\end{array}$ & $\begin{array}{c}137 . \\
3\end{array}$ \\
\hline $\mathrm{P}_{2} \mathrm{I}_{2}$ & 2.06 & 2.11 & $\begin{array}{c}2 . \\
39\end{array}$ & 2.44 & $\begin{array}{c}86 . \\
03\end{array}$ & $\begin{array}{c}86 . \\
64\end{array}$ & $\begin{array}{c}162 . \\
4\end{array}$ & $\begin{array}{c}165 . \\
4\end{array}$ \\
\hline $\mathrm{P}_{2} \mathrm{I}_{3}$ & 2.18 & 2.25 & $\begin{array}{c}2 . \\
54\end{array}$ & 2.57 & $\begin{array}{c}88 . \\
48\end{array}$ & $\begin{array}{c}89 . \\
55\end{array}$ & $\begin{array}{c}177 . \\
4\end{array}$ & $\begin{array}{c}180 . \\
5\end{array}$ \\
\hline $\mathrm{P}_{3} \mathrm{I}_{1}$ & 1.42 & 1.46 & $\begin{array}{c}1 . \\
82\end{array}$ & 1.83 & $\begin{array}{c}75 . \\
37\end{array}$ & $\begin{array}{c}76 . \\
73\end{array}$ & $\begin{array}{c}121 . \\
4\end{array}$ & $\begin{array}{c}123 . \\
5\end{array}$ \\
\hline $\mathrm{P}_{3} \mathrm{I}_{2}$ & 1.60 & 1.60 & $\begin{array}{c}1 . \\
95\end{array}$ & 1.94 & $\begin{array}{c}80 . \\
76\end{array}$ & $\begin{array}{c}81 . \\
73\end{array}$ & $\begin{array}{c}147 . \\
1\end{array}$ & $\begin{array}{c}148 . \\
4\end{array}$ \\
\hline $\mathrm{P}_{3} \mathrm{I}_{3}$ & 1.88 & 1.87 & $\begin{array}{c}2 . \\
12\end{array}$ & 2.14 & $\begin{array}{c}82 . \\
55\end{array}$ & $\begin{array}{c}83 . \\
87\end{array}$ & $\begin{array}{c}160 . \\
9\end{array}$ & $\begin{array}{c}163 . \\
9\end{array}$ \\
\hline $\mathrm{P}_{4} \mathrm{I}_{1}$ & 1.56 & 1.57 & $\begin{array}{c}1 . \\
95\end{array}$ & 1.96 & $\begin{array}{c}78 . \\
26\end{array}$ & $\begin{array}{c}79 . \\
83\end{array}$ & $\begin{array}{c}133 . \\
5\end{array}$ & $\begin{array}{c}137 . \\
0\end{array}$ \\
\hline $\mathrm{P}_{4} \mathrm{I}_{2}$ & 1.75 & 1.73 & $\begin{array}{c}2 . \\
10\end{array}$ & 2.10 & $\begin{array}{c}81 . \\
77\end{array}$ & $\begin{array}{c}82 . \\
25\end{array}$ & $\begin{array}{c}155 . \\
5\end{array}$ & $\begin{array}{c}158 . \\
0\end{array}$ \\
\hline $\mathrm{P}_{4} \mathrm{I}_{3}$ & 1.97 & 2.02 & $\begin{array}{c}2 . \\
28\end{array}$ & 2.32 & $\begin{array}{c}82 . \\
50\end{array}$ & $\begin{array}{c}83 . \\
11\end{array}$ & $\begin{array}{c}167 . \\
8\end{array}$ & $\begin{array}{c}170 . \\
6\end{array}$ \\
\hline $\mathrm{S}_{\mathrm{Em}}^{+}$ & 0.05 & 0.05 & $\begin{array}{c}0.07 \\
0\end{array}$ & 0.07 & 2.85 & 2.77 & 4.00 & 3.00 \\
\hline $\begin{array}{c}\mathrm{CD} \\
5 \%\end{array}$ & 0.15 & 0.15 & $\begin{array}{c}0 . \\
21\end{array}$ & 0.21 & 8.78 & 8.54 & $\begin{array}{c}12.2 \\
8\end{array}$ & $\begin{array}{c}10 . \\
16\end{array}$ \\
\hline
\end{tabular}

The quality parameters viz., sucrose percentage and CCS percentage were not influenced significantly among the planting methods (Table 2). While, commercial cane sugar (CCS) was significantly influenced by planting methods and $120 \mathrm{~cm}$ row spaced furrow planting with dhaincha green manure sowing at 30 DAP and mulching at 75 DAP recorded significantly higher CCS (21.64 and $22.00 \mathrm{t} / \mathrm{ha}$, respectively) as compared to others. However, it was at par with $120 \mathrm{~cm}$ row spaced furrow planting with alternate skip furrow irrigation after earthing up + dhaincha green manure mulching (21.16 and $21.54 \mathrm{t} / \mathrm{ha}$, respectively). This increased commercial cane sugar yield was mainly attributed to increased cane yield.

Table 2. Sugarcane quality parameters and total water used as influenced planting methods and irrigation schedules

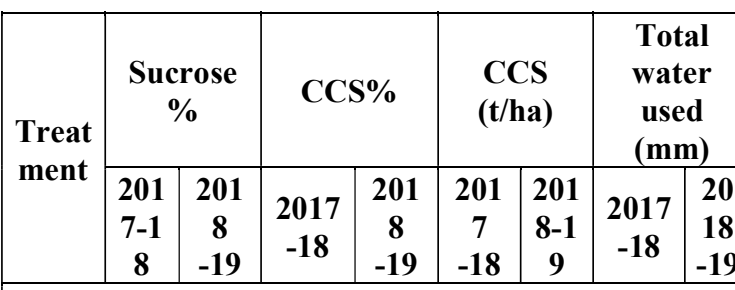

\begin{tabular}{|c|c|c|c|c|c|c|c|c|}
\hline \multicolumn{1}{|c|}{ Planting methods } \\
\hline $\mathrm{P}_{1}$ & $\begin{array}{c}18 . \\
95\end{array}$ & $\begin{array}{c}19.0 \\
4\end{array}$ & $\begin{array}{c}13.4 \\
3\end{array}$ & $\begin{array}{c}13 . \\
47\end{array}$ & 19.99 & $\begin{array}{c}20 . \\
12\end{array}$ & 2146 & $\begin{array}{c}20 \\
83\end{array}$ \\
\hline $\mathrm{P}_{2}$ & $\begin{array}{c}19 . \\
21\end{array}$ & $\begin{array}{c}19.1 \\
7\end{array}$ & $\begin{array}{c}13.6 \\
8\end{array}$ & $\begin{array}{c}13 . \\
68\end{array}$ & 21.64 & $\begin{array}{c}22 . \\
00\end{array}$ & 2059 & $\begin{array}{c}20 \\
10\end{array}$ \\
\hline $\mathrm{P}_{3}$ & $\begin{array}{c}19 . \\
41\end{array}$ & $\begin{array}{c}19.5 \\
6\end{array}$ & $\begin{array}{c}13.7 \\
6\end{array}$ & $\begin{array}{c}13 . \\
82\end{array}$ & 19.68 & $\begin{array}{c}20 . \\
05\end{array}$ & 1801 & 18 \\
28
\end{tabular}

Scheduling of irrigation

\begin{tabular}{|c|c|c|c|c|c|c|c|c|}
\hline $\mathrm{I}_{1}$ & $\begin{array}{c}19 . \\
52\end{array}$ & $\begin{array}{c}19.3 \\
3\end{array}$ & $\begin{array}{c}13.8 \\
4\end{array}$ & $\begin{array}{c}13.9 \\
1\end{array}$ & $\begin{array}{c}17.9 \\
6\end{array}$ & $\begin{array}{c}18 . \\
34\end{array}$ & 1751 & 17 \\
25 \\
$\mathrm{I}_{2}$ & $\begin{array}{c}19 . \\
27\end{array}$ & $\begin{array}{c}19.3 \\
7\end{array}$ & $\begin{array}{c}13.6 \\
9\end{array}$ & $\begin{array}{c}13.6 \\
5\end{array}$ & $\begin{array}{c}21.1 \\
2\end{array}$ & $\begin{array}{c}21 . \\
24\end{array}$ & 1955 & $\begin{array}{c}19 \\
34\end{array}$ \\
\hline $\mathrm{I}_{3}$ & $\begin{array}{c}19 . \\
03\end{array}$ & $\begin{array}{c}19.2 \\
9\end{array}$ & $\begin{array}{c}13.5 \\
5\end{array}$ & $\begin{array}{c}13.5 \\
9\end{array}$ & $\begin{array}{c}22.7 \\
7\end{array}$ & $\begin{array}{c}23 . \\
20\end{array}$ & 2189 & $\begin{array}{c}20 \\
95\end{array}$ \\
\hline $\begin{array}{c}\mathrm{S} . \mathrm{Em} . \\
\pm\end{array}$ & $\begin{array}{c}0.0 \\
8\end{array}$ & 0.10 & 0.06 & 0.06 & 0.48 & $\begin{array}{c}0.4 \\
6\end{array}$ & 15.6 & $\begin{array}{c}12 \\
1\end{array}$ \\
\hline $\begin{array}{c}\mathrm{CD} \\
\text { @ 5\% }\end{array}$ & $\mathrm{NS}$ & $\mathrm{NS}$ & $\mathrm{NS}$ & $\mathrm{NS}$ & 1.89 & $\begin{array}{c}1.8 \\
2\end{array}$ & 61.3 & $\begin{array}{c}47 \\
5\end{array}$ \\
\hline
\end{tabular}

Planting methods X Irrigation Scheduling

\begin{tabular}{|c|c|c|c|c|c|c|c|c|}
\hline $\mathrm{P}_{1} \mathrm{I}_{1}$ & $\begin{array}{l}19 . \\
02\end{array}$ & $\begin{array}{c}18.8 \\
7\end{array}$ & $\begin{array}{c}13.4 \\
4\end{array}$ & $\begin{array}{c}13.4 \\
3\end{array}$ & $\begin{array}{c}17.2 \\
5\end{array}$ & $\begin{array}{l}17 . \\
39\end{array}$ & 1881 & $\begin{array}{l}18 \\
37\end{array}$ \\
\hline $\mathrm{P}_{1} \mathrm{I}_{2}$ & $\begin{array}{l}19 . \\
03\end{array}$ & $\begin{array}{c}19.2 \\
0\end{array}$ & $\begin{array}{c}13.5 \\
0\end{array}$ & $\begin{array}{c}13.5 \\
3\end{array}$ & $\begin{array}{c}20.4 \\
8\end{array}$ & $\begin{array}{l}20 . \\
38\end{array}$ & 2171 & $\begin{array}{l}21 \\
70\end{array}$ \\
\hline $\mathrm{P}_{1} \mathrm{I}_{3}$ & $\begin{array}{c}18 . \\
81\end{array}$ & $\begin{array}{c}19.0 \\
5\end{array}$ & $\begin{array}{c}13.3 \\
6 \\
\end{array}$ & $\begin{array}{c}13.4 \\
5\end{array}$ & $\begin{array}{c}22.2 \\
2 \\
\end{array}$ & $\begin{array}{r}22 . \\
60 \\
\end{array}$ & 2386 & $\begin{array}{l}22 \\
41 \\
\end{array}$ \\
\hline $\mathrm{P}_{2} \mathrm{I}_{1}$ & $\begin{array}{r}19 . \\
62 \\
\end{array}$ & $\begin{array}{c}19.0 \\
7\end{array}$ & $\begin{array}{c}13.9 \\
6\end{array}$ & $\begin{array}{c}14.0 \\
0\end{array}$ & $\begin{array}{c}18.9 \\
1 \\
\end{array}$ & $\begin{array}{l}19 . \\
21 \\
\end{array}$ & 1845 & $\begin{array}{l}18 \\
14 \\
\end{array}$ \\
\hline $\mathrm{P}_{2} \mathrm{I}_{2}$ & $\begin{array}{r}19 . \\
11 \\
\end{array}$ & $\begin{array}{c}19.3 \\
3 \\
\end{array}$ & $\begin{array}{c}13.6 \\
3 \\
\end{array}$ & $\begin{array}{c}13.6 \\
0\end{array}$ & $\begin{array}{c}22.1 \\
5 \\
\end{array}$ & $\begin{array}{c}22 . \\
50 \\
\end{array}$ & 2057 & $\begin{array}{l}20 \\
24 \\
\end{array}$ \\
\hline $\mathrm{P}_{2} \mathrm{I}_{3}$ & $\begin{array}{l}18 . \\
89 \\
\end{array}$ & $\begin{array}{c}19.1 \\
1 \\
\end{array}$ & $\begin{array}{c}13.4 \\
5 \\
\end{array}$ & $\begin{array}{c}13.4 \\
6 \\
\end{array}$ & $\begin{array}{c}23.8 \\
5 \\
\end{array}$ & $\begin{array}{l}24 . \\
29 \\
\end{array}$ & 2277 & $\begin{array}{l}21 \\
90 \\
\end{array}$ \\
\hline $\mathrm{P}_{3} \mathrm{I}_{1}$ & $\begin{array}{r}19 . \\
67\end{array}$ & $\begin{array}{c}19.7 \\
6\end{array}$ & $\begin{array}{c}13.9 \\
1\end{array}$ & $\begin{array}{c}14.0 \\
3\end{array}$ & $\begin{array}{c}16.8 \\
9\end{array}$ & $\begin{array}{l}17 . \\
33\end{array}$ & 1683 & $\begin{array}{l}16 \\
71 \\
\end{array}$ \\
\hline $\mathrm{P}_{3} \mathrm{I}_{2}$ & $\begin{array}{l}19 . \\
23\end{array}$ & $\begin{array}{c}19.4 \\
9\end{array}$ & $\begin{array}{c}13.6 \\
1\end{array}$ & $\begin{array}{c}13.6 \\
3\end{array}$ & $\begin{array}{c}20.0 \\
2\end{array}$ & $\begin{array}{l}20 . \\
22\end{array}$ & 1877 & $\begin{array}{l}18 \\
35\end{array}$ \\
\hline
\end{tabular}




\begin{tabular}{|c|c|c|c|c|c|c|c|c|}
\hline $\mathrm{P}_{3} \mathrm{I}_{3}$ & 19. & 19.4 & 13.7 & 13.7 & 22.1 & 22. & 2083 & 19 \\
& 33 & 5 & 9 & 2 & 60 & & 77 \\
\hline $\mathrm{P}_{4} \mathrm{I}_{1}$ & $\begin{array}{c}19 . \\
77\end{array}$ & $\begin{array}{c}19.6 \\
1\end{array}$ & $\begin{array}{c}14.0 \\
6\end{array}$ & $\begin{array}{c}14.1 \\
8\end{array}$ & $\begin{array}{c}18.7 \\
7\end{array}$ & $\begin{array}{c}19 . \\
43\end{array}$ & 1593 & $\begin{array}{c}15 \\
77\end{array}$ \\
\hline $\mathrm{P}_{4} \mathrm{I}_{2}$ & $\begin{array}{c}19 . \\
71\end{array}$ & $\begin{array}{c}19.4 \\
7\end{array}$ & $\begin{array}{c}14.0 \\
4\end{array}$ & $\begin{array}{c}13.8 \\
3\end{array}$ & $\begin{array}{c}21.8 \\
4\end{array}$ & $\begin{array}{c}21 . \\
86\end{array}$ & 1717 & $\begin{array}{c}17 \\
05\end{array}$ \\
\hline $\mathrm{P}_{4} \mathrm{I}_{3}$ & $\begin{array}{c}19 . \\
11\end{array}$ & $\begin{array}{c}19.5 \\
7\end{array}$ & $\begin{array}{c}13.6 \\
3\end{array}$ & $\begin{array}{c}13.6 \\
7\end{array}$ & $\begin{array}{c}22.8 \\
7\end{array}$ & $\begin{array}{c}23 . \\
32\end{array}$ & 2010 & $\begin{array}{c}19 \\
72\end{array}$ \\
\hline $\begin{array}{c}\mathrm{S} . \mathrm{Em} . \\
\pm\end{array}$ & $\begin{array}{c}0.1 \\
2\end{array}$ & 0.22 & 0.09 & 0.07 & 0.41 & $\begin{array}{c}0.2 \\
5\end{array}$ & 29.4 & $\begin{array}{c}33 . \\
0\end{array}$ \\
\hline $\begin{array}{c}\mathrm{CD} \\
@ 5 \%\end{array}$ & $\mathrm{NS}$ & $\mathrm{NS}$ & $\mathrm{NS}$ & $\mathrm{NS}$ & 1.26 & $\begin{array}{c}0.7 \\
6\end{array}$ & 90.7 & $\begin{array}{c}10 \\
1.7\end{array}$ \\
\hline
\end{tabular}

\subsection{Irrigation schedules}

Cane yield and Yield parameters of sugarcane improved significantly with the increase in frequency of irrigation (Table 1). The crop irrigated at IW: CPE of 1.0 recorded significantly higher cane yield (168.1 and $170.8 \mathrm{t} / \mathrm{ha}$, respectively) as compared to $\mathrm{IW} / \mathrm{CPE}$ ratio of 0.60 (129.7 and $131.8 \mathrm{t} / \mathrm{ha}$, respectively). However, it was at par with 0.80 IW:CPE schedule (157.2 and $155.6 \mathrm{t} / \mathrm{ha}$, respectively). This increased yield was mainly attributed to enhanced yield attributes viz., single cane weight, cane length and number of millable canes. The enhanced millabale cane number was because of better sprouting and tillering of crop under frequent watering, which improved the microclimatic condition in the crop during. Similarly, single cane weight was also significantly higher in frequent irrigation applied at an IW: CPE schedule of 1.0 and 0.80 than 0.60 IW: CPE schedules, because of improved physiological processes. These all yield-attributing characters resulted in significantly higher cane and sugar yields in crop irrigated at an IW: CPE of 1 and 0.80 than IW: CPE schedules of 0.60 [5] also reported significant increase in cane yield with frequent irrigation at IW/CPE of 0.8 over IW/CPE of 0.6 owing to improved uptake of water with dissolved nutrients in frequently irrigated crop. Juice-quality traits like sucrose content and CCS remained statistically at par among all the 3 irrigation schedules. Total water applied was lowest in IW/CPE of 0.60 (1751 and 1725 $\mathrm{mm}$, respectively) during both the years of experimentation (Table 2). While, IW/CPE of 1.0 used highest amount of water (2189 and $2095 \mathrm{~mm}$, respectively).

\section{CONCLUSIONS}

The scheduling of irrigation with mulch under different planting methods had significant influence on sugarcane yield. The best was observed in $120 \mathrm{~cm}$ row spaced furrow planting with alternate skip furrow irrigation after earthing up + dhaincha green manure mulching and it was found to enhance the water use efficiency and gave at par yield as that of $120 \mathrm{~cm}$ row spaced furrow planting with dhaincha green manure with all furrow irrigation.

\section{References}

1. S. Akhtar, A. Wahid, M. Akram and E. Rausl. Some growth, photosynthetic and anatomical attributes of sugarcane genotypes under $\mathrm{NaCl}$ salinity International Journal of Agricultural Biology, 3(4) 439-443 (2001)

2. S. A. Enan. Effect of transplanting and soil application of boron and zinc on yield and quality of sugar beet Ph. D. Thesis Agronomy Department Faculty of Agriculture Al - Azhar University, Egypt (2004)

3. D.G. Hapase, B.B. Gunjal and A.S. Deshmukh, (In:) Irrigation management for sugarcane Recent Advances in Sugarcane, Rao, P.N. (Editor), K.C.P. Ltd, Vuyyru, Andhra Pradesh, India, pp 248-70 (1990)

4. P. Jamuna, K.R. Swamy, K.V. Rao and Y. Satyanarayana. Ground water quality assessment with reference to sugarcane cultivation in Chelluru and Pithapuram factory areas of Andhra Pradesh Cooperative Sugar, 26(1) 105-9 (1994)

5. Kumar, Shiv and S.N.L. Srivastava. Response of sugarcane to alternate furrow irrigation and trash mulch Indian Journal of Sugarcane Technology, 6(1) 69-73 (1991)

6. Lal, Menhi and S.K. Shukla. (In:) 50 Years of Sugarcane Research in India Production practices for sugarcane. H.N. Shahi, A.K. Srivastava, and O.K. Sinha, (Editors). ICAR-Indian Institute of Sugarcane Research, Lucknow 226 002. pp 131-52 (2000)

7. S.K. Sastry and A.V. Chari (In:) Proceedings 4th Biennial Conference Sugarcane Research and Development Workers, India pp 145-59 (1960)

8. P.N. Singh, S.K. Shukla and V.K. Bhatnagar, Optimizing soil moisture regime to increase water use efficiency of sugarcane (Saccharum spp. hybrid complex) in subtropical India Agricultural Water Management, 90(1-2) 95-100 (2007)

9. Singh, Ishwar. Optimizing irrigation schedule in sugarcane (Saccharum spp. hybrid complex) under different planting methods in sub-tropical India Indian Journal of Sugarcane Technology, 27(2) 68-72 (2012)

10. P.N. Singh and S.C. Mohan. Water use and yield re-response of sugarcane under different irrigation schedules and nitrogen levels in a subtropical region Agricultural Water Management, 26(4) 253-64 (1994)

11. D.F. Uwah and G.A. Iwo. Effectiveness of organic mulch on the productivity of maize (Zea mays L.) and weed growth The Journal of Animal and Plant Sciences, 21(3) 525-530 (2011) 